\title{
Association of lipid-related genes implicated in conceptus elongation with female fertility traits in dairy cattle
}

\author{
Rostam Abdollahi-Arpanahi, ${ }^{1}$ Murilo R. Carvalho, ${ }^{2}$ Eduardo S. Ribeiro, ${ }^{2 *}$ and Francisco Peñagaricano ${ }^{1,3 *}$ \\ ${ }^{1}$ Department of Animal Sciences, University of Florida, Gainesville 32611 \\ ${ }^{2}$ Department of Animal Biosciences, University of Guelph, Guelph, ON N1G 2W1, Canada \\ ${ }^{3}$ University of Florida Genetics Institute, University of Florida, Gainesville 32611
}

\section{ABSTRACT}

Elongation of the preimplantation conceptus is a requirement for pregnancy success in ruminants, and failures in this process are highly associated with subfertility in dairy cattle. Identifying genetic markers that are related to early conceptus development and survival and utilizing these markers in selective breeding can improve the reproductive efficiency of dairy herds. Here, we evaluated the association of 1,679 SNP markers within or close to 183 candidate genes involved in lipid metabolism of the elongating conceptus with different fertility traits in US Holstein cattle. A total of 27,371 bulls with predicted transmitting ability records for daughter pregnancy rate, cow conception rate, and heifer conception rate were used as the discovery population. The associations found in the discovery population were validated using 2 female populations (1,122 heifers and 2,138 lactating cows) each with 4 fertility traits, including success to first insemination, number of services per conception, age at first conception for heifers, or days open for cows. Marker effects were estimated using a linear mixed model with SNP genotype as a linear covariate and a random polygenic effect. After multiple testing correction, 39 SNP flagging 27 candidate genes were associated with at least one fertility trait in the discovery population. Of these 39 markers, 3 SNP were validated in the heifer population and 4 SNP were validated in the cow population. The $3 \mathrm{SNP}$ validated in heifers are located within or near genes CAT, MYOF, and RBP4, and the $4 \mathrm{SNP}$ validated in lactating cows are located within or close to genes CHKA, GNAI1, and HMOX2. These validated genes seem to be relevant for reducing pregnancy losses, and the SNP within these genes are excellent

\footnotetext{
Received June 5, 2019.

Accepted July 12, 2019.

*Corresponding authors: eribeiro@uoguelph.ca and fpenagaricano@
} ufl.edu candidates for inclusion in genomic tests to improve reproductive performance in dairy cattle.

Key words: candidate genes, conceptus development, reproductive traits, validation study

\section{INTRODUCTION}

Elongation of the preimplantation conceptus (embryo plus extraembryonic membranes) is essential for pregnancy success in ruminants and is highly associated with pregnancy failures in dairy cattle. For instance, it has been estimated that $39 \%$ of viable blastocysts on Day 6 of development fail to elongate and maintain pregnancy by d 28 of gestation (Ribeiro, 2018). Embryonic losses during this period are substantial and cause considerable economic losses, waste of resources, and reduction in the sustainability of dairy cattle production (Ribeiro et al., 2012). Likewise, future improvements in reproductive efficiency in dairy cattle herds will require the development of strategies to minimize those embryonic losses. One option to improve reproduction efficiency is to identify genetic markers associated with early embryonic development and using them in marker-assisted selection.

To prevent embryonic losses using selection, genetic variability should exist. Estimates of heritability for superovulation records (number of structures recovered and number of good quality embryos) ranged from 0.15 to 0.27 , for in vitro fertilization data (number of oocytes collected, number of cleaved embryos, number of high- and low-quality embryos, and number of transferrable embryos) varied from 0.01 to 0.21 , and for pregnancy success after embryo transfer ranged from 0.02 to 0.03 (Parker Gaddis et al., 2017). According to these heritability estimates, genetic improvement for traits related to embryonic development and survival is possible. It should be noted that none of these traits are officially recorded in dairy cattle breeding programs. On the other hand, some related traits, such as daughter pregnancy rate (DPR), cow conception rate $(\mathbf{C C R})$, and heifer conception rate $(\mathbf{H C R})$, are 
routinely evaluated and included in breeding goals and selection programs. Hence, the question is whether genes relevant to conceptus development are associated with officially published fertility traits in dairy cattle.

To date, genomic regions associated with fertility traits have been found for calving to first service interval (Höglund et al., 2009; Sahana et al., 2010), interval from first service to last service (Höglund et al., 2009), days open (Schulman et al., 2008; Ortega et al., 2017), cow nonreturn rate (Höglund et al., 2009; Olsen et al., 2011), heifer nonreturn rate (Holmberg and AnderssonEklund, 2006), daughter pregnancy rate (Cole et al., 2011; Cochran et al., 2013; Ortega et al., 2016), sire conception rate (Peñagaricano et al., 2012; Han and Peñagaricano, 2016), and HCR and CCR (Cochran et al., 2013; Ortega et al., 2016). These genomic regions generally explain only a small proportion of the genetic variance underlying reproductive performance.

Recently, Ribeiro et al. (2016b) carried out a transcriptomic analysis from conceptuses collected during the onset of elongation and found that lipid metabolism was one of the top molecular and cellular functions associated with conceptus elongation. Hence, the identification and subsequent use of SNP markers within these genes involved in lipid metabolism might improve reliability of genomic predictions for fertility traits. Therefore, the main goal of this study was to evaluate the association between markers within or around lipid-related genes implicated in conceptus elongation and reproductive performance. Three female fertility traits-DPR, HCR, and CCR - were evaluated in a large population of Holstein bulls. Two female populations, one consisting of heifers and another of lactating cows, were used to validate the associations revealed in the discovery (bull) population. Each female population (validation) had 4 fertility traits, which include success or fail at first insemination (SF), number of services per conception (NSC), fertility category (FertC), and age at first conception (AFC) for heifers or days open (DO) for cows.

\section{MATERIALS AND METHODS}

\section{Candidate Genes}

In a previous gene expression study (Ribeiro et al., 2016b), transcervical uterine flushings of single-ovulating lactating dairy cows $(\mathrm{n}=160)$ on d 15 after AI resulted in the recovery of preimplantation conceptuses $(\mathrm{n}=65)$ in different stages of elongation, ranging from 1 to $60 \mathrm{~mm}$ in length. Recovered conceptuses were categorized based on length as ovoid (1-4 mm), tubular (5-19 $\mathrm{mm})$, or filamentous (20-60 $\mathrm{mm})$, and total RNA was extracted from individual conceptuses. Good qual- ity RNA (integrity number $>7.5$ ) from a subsample of conceptuses from each group (ovoid $=8$; tubular $=17$; and filamentous $=17$ ) was subjected to transcriptomic analysis using the GeneChip Bovine Genome Array (Affymetrix, Santa Clara, CA). In total, 1,611 transcripts were differentially expressed in at least 1 of the 3 pairwise comparisons among experimental groups, which revealed important biological events associated with the onset of elongation of the preimplantation conceptus.

Pathway and functional analyses of the differentially expressed transcripts revealed that lipid metabolism was one of the top molecular and cellular functions associated with conceptus elongation (Ribeiro et al., 2016b). Specifically, 194 genes found to be related directly or indirectly to lipid metabolism and associated with elongation of bovine conceptus were used as candidate genes in this study (Supplementary File S1; https://doi.org/10.3168/jds.2019-17068). The details of sampling, nucleic acid isolation, and analysis of transcriptome from conceptuses cells is described in Ribeiro et al. (2016b). The annotation information of candidate genes was obtained from the Ensembl database using the R package "biomaRt" (Durinck et al., 2009).

\section{Discovery Population}

To further investigate the results of the microarray study using a genome-wide approach, a total of 27,371 Holstein bulls with PTA records for HCR, CCR, and DPR were used as the discovery population. The trait $\mathrm{HCR}$ is defined as the percentage of inseminated heifers that become pregnant at each service (heifer's ability to conceive); CCR is defined as the percentage of inseminated cows that become pregnant at each service (lactating cow's ability to conceive); and DPR is defined as the percentage of nonpregnant cows that become pregnant during each 21-d period (a cow's overall ability to start cycling again, show heat, conceive, and maintain a pregnancy). The Holstein bulls' PTA records and corresponding reliabilities were retrieved from July 2018 US national genetic evaluation from the Council on Dairy Cattle Breeding website (https: //www.uscdcb.com). The mean reliabilities for DPR, CCR, and HCR were $0.83,0.82$, and 0.74 , respectively; all bulls had reliability values $>0.70$ for the 3 traits. The descriptive statistics of these traits are shown in Table 1.

\section{Validation Populations}

Two Holstein female populations consisted of 1,122 heifers and 2,138 lactating cows from a large commercial farm in Florida were used as validation populations 
ABDOLLAHI-ARPANAHI ET AL.

Table 1. Descriptive statistics of fertility traits in the discovery and validation Holstein populations

\begin{tabular}{|c|c|c|c|c|c|}
\hline Population and trait ${ }^{1}$ & $\mathrm{~N}$ & Mean & SD & Minimum & Maximum \\
\hline \multicolumn{6}{|l|}{ Discovery: Bulls } \\
\hline DPR (DEBV) & 27,371 & -3.09 & 20.403 & -81.9 & 80.91 \\
\hline CCR (DEBV) & 27,371 & -0.31 & 25.25 & -101.5 & 100.3 \\
\hline HCR (DEBV) & 27,371 & 4.61 & 19.17 & -76.65 & 82.27 \\
\hline \multicolumn{6}{|l|}{ Validation: Heifers } \\
\hline $\mathrm{SF}(\%)$ & 1,126 & 55 & 49 & 0 & 1 \\
\hline NSC (no.) & 1,122 & 2.18 & 1.92 & 1 & 12 \\
\hline $\operatorname{AFC}(d)$ & 1,122 & 428.38 & 58.22 & 358 & 786 \\
\hline FertC & 1,126 & 1.62 & 0.76 & 1 & 4 \\
\hline \multicolumn{6}{|l|}{ Validation: Cows } \\
\hline $\mathrm{SF}(\%)$ & 2,346 & 39 & 49 & 0 & 1 \\
\hline NSC (no.) & 2,138 & 2.32 & 1.69 & 1 & 9 \\
\hline $\mathrm{DO}(\mathrm{d})$ & 2,138 & 122.49 & 55.58 & 47 & 303 \\
\hline FertC & 2,346 & 1.93 & 0.92 & 1 & 4 \\
\hline
\end{tabular}

${ }^{1} \mathrm{DPR}$, daughter pregnancy rate; DEBV, deregressed breeding values; CCR, cow conception rate; HCR, heifer conception rate; SF, success at first insemination; NSC, number of services per conception; AFC, age at first conception; FertC, fertility category; DO, days open.

(calving date: from 2012 to 2017). Information related to reproductive performance (date and success of breedings) was retrieved from the herd management software (PCDART, Dairy Records Management Systems, Raleigh, NC). Pregnancy diagnosis of all cows and heifers was performed by rectal palpation and ultrasonography examination of the uterus $45 \pm 3 \mathrm{~d}$ after breeding. Four reproductive traits, including SF, NSC, FertC, and AFC (heifers) or DO (cows), were used to validate the findings obtained in the discovery population. The trait $\mathrm{SF}$ was coded as $\mathrm{SF}=1$ if the heifer/cow became pregnant at the first insemination, otherwise $\mathrm{SF}=0$. The trait DO was computed as the interval between the previous calving date and the date of the breeding that resulted in a positive pregnancy diagnosis. The minimum and maximum of DO after data editing was 47 and 303, respectively. Fertility categorization was based on pregnancy success after breeding as follow: $1=$ pregnant at the first breeding; $2=$ pregnant at the second or third breeding; $3=$ pregnant at fourth or more breeding; and $4=$ fail to become pregnant. Pregnancy status for FertC was based on diagnosis at $45 \mathrm{~d}$ after breeding.

\section{Genotype Data}

Genome-wide data for 312,614 SNP markers were available for the 27,371 Holstein bulls. The SNP data were kindly provided by the Cooperative Dairy DNA Repository (CDDR; Columbia, MO). This set of SNP markers is available in the BovineHD Genotyping BeadChip (Illumina Inc., San Diego, CA; Wiggans et al., 2016). Of the 194 differentially expressed genes involved in lipid metabolism, 183 were marked by $1,708 \mathrm{SNP}$ in the $312 \mathrm{k}$ SNP panel. These SNP were located either within the candidate genes (i.e., genomic sequence between the start of the first exon and the end of the last exon) or at most $10 \mathrm{~kb}$ upstream or downstream of the candidate genes. The distance of 10 $\mathrm{kb}$ was used to capture proximal regulatory and other functional regions that may lie outside, but close to, the candidate genes. The validation populations were genotyped with 60,671 SNP markers. Of 1,708 candidate SNP, 208 SNP were found in the 60k panel and the remaining 1,500 SNP were imputed using the $312 \mathrm{k}$ panel with the software "FImpute" (Sargolzaei et al., 2014). After quality control of candidate SNP for minor allele frequency (exclusion of SNP with minor allele frequency <0.01) in both male and female populations, 1,679 SNP remained for the association study.

\section{Statistical Analysis}

Discovery Phase. The association analyses between the 1,679 SNP and the fertility traits were conducted using a mixed linear model. Because a bull's PTA includes both pedigree information and daughter phenotypic performance, there is a risk that SNP would be associated on the basis of parent average rather than own performance. Therefore, deregressed Holstein bull proofs (DEBV) were computed as described in Nayeri et al. (2017). To assess the significance of each SNP, the following linear mixed model was used:

$$
\mathbf{y}=1 \mu+\mathbf{X b}+\mathbf{Z u}+\mathbf{e},
$$

where $\mathbf{y}$ is the vector of DEBV, $\mu$ is the intercept, $\mathbf{b}$ is the vector of SNP substitution effects, $\mathbf{u}$ is the vector of random polygenic effects, $\mathbf{e}$ is the vector of random residual effects, $\mathbf{X}$ is the genotype matrix relating DEBV 
and SNP genotypes coded as 0,1 , and 2 for $B B, A B$, and $A A$, respectively, and $\mathbf{Z}$ is a design matrix relating bulls to observations. The 2 random effects $\mathbf{u}$ and $\mathbf{e}$ were distributed as $\mathbf{u} \sim N\left(0, \mathbf{G} \sigma_{a}^{2}\right)$ and $\mathbf{e} \sim N\left(0, \mathbf{R}^{-1} \sigma_{e}^{2}\right)$, where $\sigma_{a}^{2}$ and $\sigma_{e}^{2}$ are the genomic and residual variances, respectively, $\mathbf{G}$ is the genomic relationship matrix constructed using roughly 300k SNP markers across the genome, and $\mathbf{R}$ is a diagonal matrix with its elements representing reliabilities of PTA records. Given that SNP within genes are not fully independent because of linkage disequilibrium, Bonferroni correction was performed based on the number of candidate genes (183) instead of total number of SNP. A $P$-value $\leq 0.0003$ $(0.05 / 183)$ was used as threshold for denoting significant associations in the discovery population. These analyses were carried out using the software SNP1101 tool (Sargolzaei, 2014).

Validation Phase. For SF, NSC, AFC, and FertC in heifers, the statistical model included year-season of first insemination and age at first service as fixed effects. Similarly, for SF, NSC, DO, and FertC in cows, the year-season of calving, parity, and clinical disease status in the first $21 \mathrm{~d}$ postpartum were considered fixed effects. To control for population stratification, a genomic relationship matrix was constructed using roughly 60k SNP markers across the genome and fed to the model to fit the polygenic effect. All of these association analyses were also performed using the SNP1101 tool. Given the stringent criterion used in the discovery population, a threshold of $P$-value $\leq 0.05$ was used in the validation study. Therefore, if a SNP showed a significant association in the discovery population $(P$-value $\leq 0.0003)$ and showed a significant association $(P$-value $\leq 0.05)$ with the same effect direction in any of the validation populations, then the candidate gene marked by the SNP was considered to be associated with reproductive performance.

\section{RESULTS}

\section{Discovery Population}

Thirty-nine SNP located within or close to 27 different genes (ACSS2, APC, APP, ATP9A, B4GALT1, BAX, CAT, CERS4, CHKA, CUX1, EPHX2, FNBP1, GNAI1, GPAM, GPAT3, HLF, HMOX2, LPCAT2, LPCAT4, MYOF, NCOR1, PEMT, PLA2G7, RBP4, SLC27A6, SSFA2, and TGM2) were associated (Pvalue $\leq 0.0003$ ) with at least one fertility trait in the discovery population after multiple testing correction (Figure 1; Supplemental File S1; https://doi.org/10 $.3168 /$ jds.2019-17068). A total of 11 SNPs were associated with DPR, 20 were associated with CCR, and 21 were associated with HCR.
The 11 SNP associated with DPR are within or close to 8 genes: APP, CERS4 (2 SNP), B4GALT1, FNBP1, TGM2, BAX, HMOX2 (2 SNP), and CHKA (2 SNP). The 20 SNP significantly associated with CCR are located inside or at most $10 \mathrm{~kb}$ apart from 15 genes: APP, GNAI1, SLC27A6, B4GALT1, APC, TGM2, ATP9A, LPCAT2, BAX, PLA2G7, HMOX2 (4 SNP), CUX1, RBP4, GPAM, and CHKA (3 SNP). The 21 SNP related to HCR are within or near 16 genes: SSFA2, GPAT3, SLC27A6, EPHX2, LPCAT4, ACSS2 (2 SNP), TGM2, CAT, LPCAT2, HLF (2 SNP), NCOR1 (2 SNP), PEMT, HMOX2, CUX1, MYOF, and $R B P 4$ (3 SNP).

Two SNP, rs137538530 and rs137538530, located on BTA13 and BTA25, were associated with DPR, CCR, and HCR. These SNP are located within the TGM2 and HMOX2 genes, respectively. Because DPR and CCR are highly correlated traits, of the 11 SNP associated with DPR, 8 were also associated with CCR with the same SNP effect direction as for DPR. Two SNP were associated with both DPR and HCR (rs137538530 and rs137538530), and 5 SNP (rs137538530, rs109674646, rs109164874, rs110852551, rs42672508) were associated with CCR and HCR. In all cases, the allele substitution effects were in the same direction for DPR and either HCR or CCR (Supplemental File S1; https://doi.org/ 10.3168/jds.2019-17068).

\section{Heifer Validation Population}

The estimates of genomic heritability in the heifer population for SF, NSC, AFC, and FertC were $0.010 \pm$ $0.007,0.030 \pm 0.010,0.040 \pm 0.011$, and $0.020 \pm 0.005$, respectively $( \pm \mathrm{SEM})$. The correlations between SNP effects and the number of common SNP among fertility traits between discovery and validation data sets are given in Table 2. The correlation of SNP solutions between fertility traits ranged from -0.05 (between FertC and HCR) to 0.11 (between NSC and DPR). Of the 21 SNP significantly associated with HCR in the discovery population, $3 \mathrm{SNP}$ within or near the CAT, MYOF, and $R B P 4$ genes were significantly $(P \leq 0.05)$ related to either NSC, SF, or FertC in the heifer population (Table 3). The direction of the SNP substitution effects in the heifer population supported the findings obtained in the bull population; hence, these 3 SNP were considered to be validated (Table 3; Supplemental File S1; https://doi.org/10.3168/jds.2019-17068).

Interestingly, the SNP on BTA15 at position $65.8 \mathrm{Mb}$ in $C A T$ was significantly associated with all 3 fertility traits in the discovery population and was also related to NSC $(P=0.04)$ and tended to be associated ( $P$ $\leq 0.10$ ) with AFC and FertC (Table 3). The SNP on BTA26 at position $14.8 \mathrm{Mb}$ in MYOF was associated 


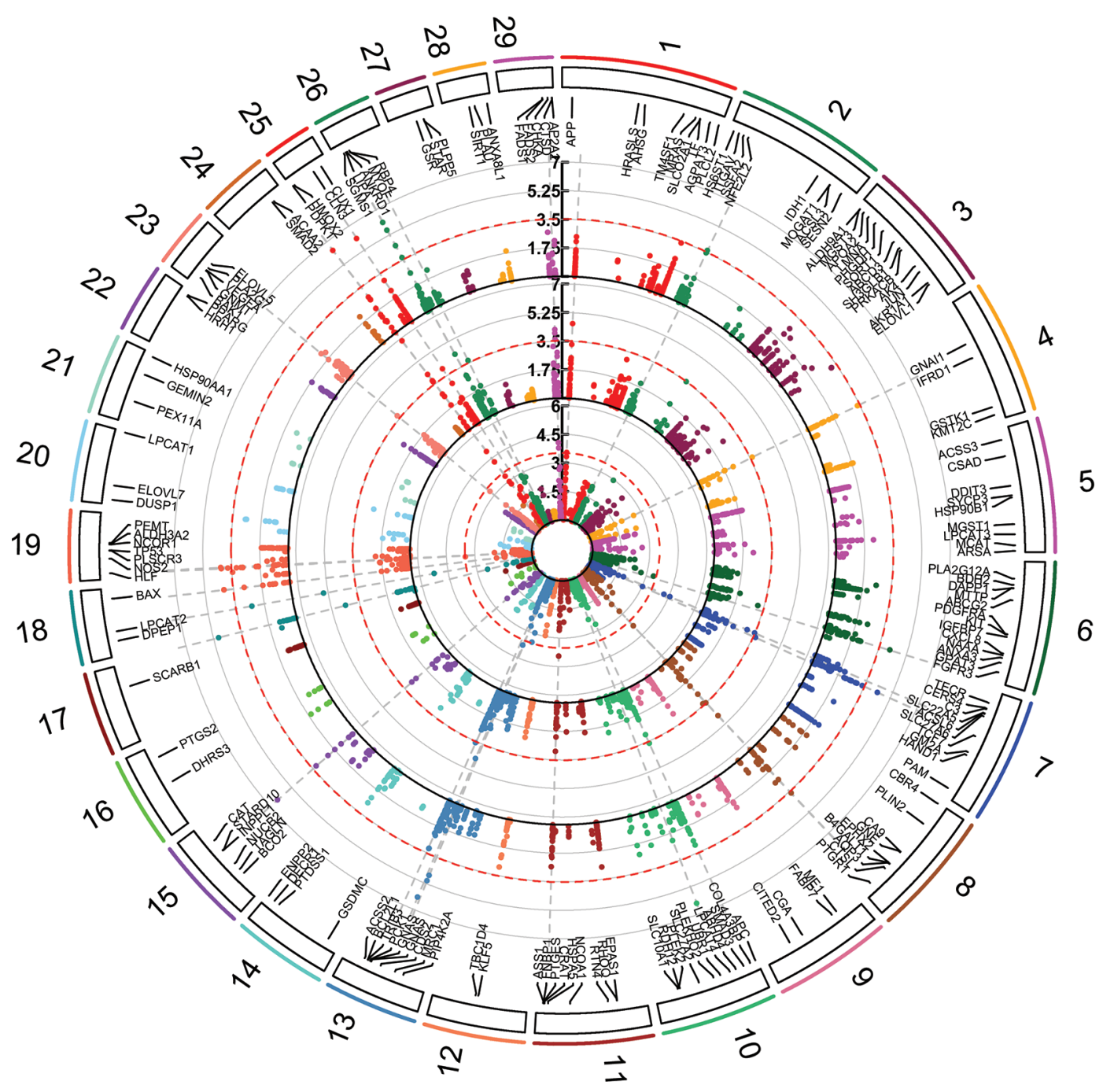

Figure 1. Circular Manhattan plot of the SNP investigated. The 3 circles, from inside to outside, represent daughter pregnancy rate, cow conception rate, and heifer conception rate, respectively. Labels in the outer circle correspond to gene names; $\mathrm{x}$-axis = genomic position of SNP in the genome; $\mathrm{y}$-axis $=$ statistical significance. The red dashed lines indicate the threshold for statistical significance.

Table 2. Number of significant SNP (diagonal; bold), correlations between SNP solutions (above the diagonal), and number of common significant SNP (below diagonal) for reproduction traits in the discovery and validation populations [heifers $(\mathrm{H})$ or cows $(\mathrm{C})$ ]

\begin{tabular}{|c|c|c|c|c|c|c|c|c|c|c|c|}
\hline Trait $^{1}$ & DPR & CCR & HCR & SF_H & NSC_H & AFC_H & FertC_H & SF_C & NSC_C & DO_C & FertC_C \\
\hline CCR & 8 & 20 & 0.57 & -0.02 & 0.09 & 0.06 & 0.04 & -0.07 & 0.09 & 0.12 & 0.09 \\
\hline HCR & 2 & 5 & 21 & 0.04 & 0.01 & -0.02 & -0.05 & -0.06 & 0.06 & 0.08 & 0.07 \\
\hline NSC_H & 0 & 0 & 1 & 38 & 89 & 0.85 & 0.84 & -0.39 & 0.29 & 0.38 & 0.41 \\
\hline AFC_H & 0 & 0 & 0 & 24 & 50 & 101 & 0.92 & -0.43 & 0.43 & 0.55 & 0.51 \\
\hline FertC_H & 0 & 1 & 2 & 56 & 45 & 30 & 79 & -0.36 & 0.33 & 0.42 & 0.41 \\
\hline FertC_C & 0 & 1 & 2 & 3 & 5 & 17 & 2 & 74 & 67 & 22 & 106 \\
\hline
\end{tabular}

DPR, daughter pregnancy rate; CCR, cow conception rate; HCR, heifer conception rate; SF, success at first insemination; NSC, number of services per conception; AFC, age at first conception; FertC, fertility category; DO, days open. 


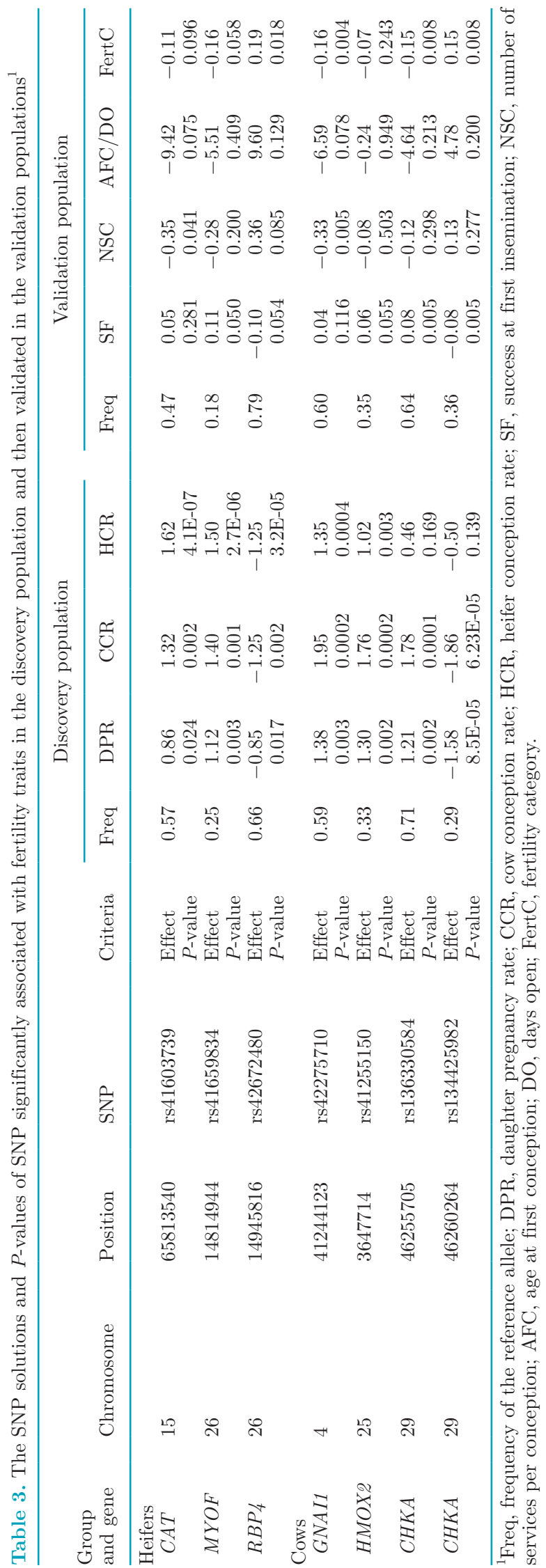

$(P=0.05)$ with $\mathrm{SF}$ and tended to be associated $(P$ $=0.06)$ with FertC (Table 3). The SNP on BTA26 at position $14.9 \mathrm{Mb}$ in $R B P 4$ was associated $(P \leq 0.05)$ with SF and FertC (Table 3).

\section{Cow Validation Population}

The estimates of genomic heritability in the cow population for SF, NSC, DO, and FertC were $0.010 \pm$ $0.005,0.040 \pm 0.016,0.030 \pm 0.011$, and $0.030 \pm 0.011$, respectively. The correlation of SNP solutions between fertility traits in the bull population and fertility traits in the cow population ranged from -0.07 (between CCR and SF) to 0.12 (between CCR and DO). Out of 39 significant SNP in the discovery population, 4 SNP within the GNAI1, HMOX2, and CHKA genes were significantly associated with at least one of the fertility traits in the cow population (Table 3 ). These SNP are located on chromosomes 4, 25, and 29. The substitution effects of these SNP were in the expected direction based on the SNP effects observed in the discovery population.

The SNP on BTA4 at position $41.2 \mathrm{Mb}$ within GNAI1 was significantly associated with CCR and HCR in the bull population and was also associated with NSC and FertC $(P \leq 0.02)$ and tended to be associated $(P=$ 0.08 ) with DO in the cow population (Table 3$)$. The SNP on BTA25 at $36.5 \mathrm{Mb}$ within HMOX2 was associated with $\mathrm{SF}(P=0.05$; Table 3$)$. Moreover, $2 \mathrm{SNP}$ within CHKA, located on BTA29 at $46.2 \mathrm{Mb}$, were significantly associated $(P=0.02)$ with $\mathrm{SF}$ and showed marginal associations $(P=0.07)$ with FertC (Table 3$)$.

\section{DISCUSSION}

Conception rate in dairy cows, measured as the proportion of cows diagnosed pregnant after a breeding, has remained low and stagnant for many years in North American herds, averaging between 30 and 35\% (Ribeiro, 2018). The reduced efficiency in pregnancy establishment after breeding is mainly explained by a high incidence of early pregnancy losses, as the rate of synchronous ovulation and fertilization of oocytes after breeding is relatively high, above $70 \%$ (Ribeiro, 2018). Thus, development of strategies to minimize the occurrence of early pregnancy losses has great potential to improve reproductive efficiency of lactating cows, which is critical for profitability and sustainability of dairy production worldwide (Ribeiro et al., 2012). A conceivably small but relevant portion of the variation in pregnancy success is explained by the genetics of the cow, the genetics of the breeding sire, and the resulting genetics of the embryo (Butler, 2013; Cole et al., 
2016; Han and Peñagaricano, 2016; Ortega et al., 2016; Ribeiro, 2018).

Using a large population of Holstein bulls as our discovery population, we were able to identify 1,679 SNP located within or close to 183 of 194 candidate genes. Our candidate genes were all associated with lipid metabolism of conceptus cells, in which transcript expression increased $(\mathrm{n}=118$ genes; range: 50 to $351 \%$ increase in expression) or decreased $(\mathrm{n}=76$ genes; range: 50 to $143 \%$ decrease in expression) during the onset of elongation of the bovine conceptus; that is, in the transition from ovoid to tubular and from tubular to filamentous (Ribeiro et al., 2016b). All sires in our discovery population had reliabilities greater than $70 \%$ for PTA of HCR, CCR, and DPR. These 3 traits are routinely evaluated in US dairy cattle and included in all merit indices (VanRaden et al., 2018). Combining the information of candidate SNP and PTA records in the bull (discovery) population, we were able to identify 39 SNP significantly associated with at least 1 of the 3 fertility traits. These SNP were within or around 27 genes likely relevant for pregnancy establishment and maintenance, and they represent polymorphisms likely important for fertility in Holsteins.

To validate the findings obtained in the discovery population, we evaluated the 39 SNP in a population of Holstein lactating cows and in a population of Holstein heifers. We collected phenotypic information on 4 fertility traits in the lactating cow population (SF, DO, NSC, FertC) and 4 fertility traits in the heifer population (SF, AFC, NSC, and FertC). All of these fertility traits are economically relevant and commonly measured in commercial herds, and they are all associated with pregnancy success. Moreover, for lactating cows, we collected detailed information related to incidence of clinical diseases postpartum, which have a strong influence on subsequent reproductive performance (Ribeiro and Carvalho, 2017). Inclusion of health postpartum in our models helped to explain the variability in fertility phenotypes and likely enhanced our ability to identify genetic markers truly associated with inherited fertility in lactating cows.

Out of the 39 SNP found to be associated with fertility traits in the sire population, 7 SNP located within or close to 6 genes were validated in lactating cows (CHKA, GNAI1, HMOX2) or heifers (CAT, MYOF, $R B P 4)$. Interestingly, all validated genes had intermediate to high transcript expression in preimplantation conceptus cells, and their expression increased 150 to $321 \%$ during the onset of elongation, in the transition from ovoid to tubular and from tubular to filamentous (Ribeiro et al., 2016b). Two of the validated SNP were located close to each other and within the CHKA gene located on BTA29. The remaining genes had one validated SNP each. The SNP rs41255150 in HMOX2 is a synonymous variant, whereas the other 6 SNP are intronic variants.

Choline kinase $\alpha(\mathrm{CHKA})$ is a mammalian enzyme that catalyzes the phosphorylation of choline to phosphocholine to produce the major membrane phospholipid phosphatidylcholine. Wu et al. (2008) generated a mouse lacking CHKA and concluded that CHKA is an essential gene for early embryonic growth. Transcript expression of $C H K A$ in bovine conceptus cells increased 2.8-fold with the onset of elongation (Ribeiro et al., 2016b) and is likely critical for the rapid synthesis of phosphatidylcholine and cell biomass required by the elongating conceptus. Sahana et al. (2010) identified a genomic region on BTA29 at position $46.9-51.9 \mathrm{Mb}$, close to CHKA, that had significant associations with number of inseminations, interval from first to last insemination, and fertility index in Holstein dairy cattle. Moreover, Frischknecht et al. (2017) detected a significant SNP on BTA29, approximately $1 \mathrm{Mb}$ downstream of $C H K A$, that was associated with days to first service in Brown Swiss cattle. These studies corroborate our results, in which the 2 validated SNP in CHKA were highly associated with DPR and CCR in the bull population and with SF and FertC in the lactating cow population.

Heterotrimeric G proteins are composed of subunits $\alpha, \beta$, and $\gamma$, and are responsible for transducing signals from $G$ protein-coupled receptors in the plasma membranes to second messengers in the cytoplasm, thus having a wide variety of functions in multiple tissues (Álvarez et al., 2015). The GNAI1 gene (G-protein subunit alpha I1) encodes one of the $\alpha$ subunits from the Gai family, which is known for its inhibitory effect on adenylyl cyclase activity. Multiple reproductive hormones, including neuropeptides, gonadotropins, and prostaglandins, bind to $\mathrm{G}$ protein-coupled receptors in target tissues and, therefore, use $\mathrm{G}$ proteins for downstream signaling. Recently, GNAI1 was also associated with progestogen-associated networks involved in ovulation (Yang et al., 2018). Moreover, de novo mutations in GNAI1 have been associated with developmental disorders in humans (Deciphering Developmental Disorders Study, 2017). In our study, the validated SNP located in GNAI1 was highly associated with all 3 fertility traits in the sire population (DPR, CCR, and HCR), and was associated with NSC, FertC, and DO in the lactating cow population. Interestingly, the peak on BTA4 at $41.2 \mathrm{Mb}$ within the GNAI1 gene identified in our study overlaps with significant regions reported for number of inseminations per conception in Nordic Holstein, Danish Jersey, and Nordic Red cattle 
(Höglund et al., 2014), conception rate in Chinese and Nordic Holsteins (Liu et al., 2017), and pregnancy rate in Brahman heifers (Porto-Neto et al., 2015).

On BTA25, our study detected a synonymous SNP at $3.65 \mathrm{Mb}$ near HMOX2; this marker was associated with all 3 fertility traits evaluated in Holstein bulls (DPR, CCR, and HCR) and with SF in lactating cows. Frischknecht et al. (2017) also reported a SNP on BTA25 at $1.5 \mathrm{Mb}$, associated with days to first service and interval between first and last insemination in Brown Swiss cattle. Heme oxygenase 2 is 1 of 2 heme oxygenases (HMOX1 and HMOX2) responsible for the degradation of heme into biliverdin, iron, and carbon monoxide, and it is involved in sensing of oxygen levels and regulation of oxidative stress in multiple tissues (Muñoz-Sánchez and Chánez-Cárdenas, 2014). Whereas HMOX2 is considered to be constitutively expressed in cells, HMOX1 is considered to be an inducible protein. In preimplantation bovine conceptuses, expression of HMOX2 was 3.3-fold greater than expression of HMOX1 (Ribeiro et al., 2016b). Heme is a prosthetic group in multiple hemeproteins such as cyclooxygenases, peroxidases, and catalases, which are critical for conceptus development and lipid metabolism (Ribeiro et al., 2016a). Nonetheless, excessive levels of free heme in the tissue environment can cause cellular damage (Kumar and Bandyopadhyay, 2005). Therefore, expression and function of heme oxygenases are critical to maintaining adequate levels of heme in the tissues and hemeprotein function.

A SNP located at $65.8 \mathrm{Mb}$ on BTA15 was associated with HCR and CCR in the sire population and with NSC, AFC, and FertC in the heifer population. This SNP is within the CAT gene. Normally, the body is protected by a wide range of antioxidant systems acting in concert with intracellular enzymes such as catalase (CAT), which remove superoxides and peroxides before they react with metal catalysts to form more reactive compounds (Miller et al., 1993). During elongation of the preimplantation conceptus, the rapid proliferation and intense metabolism of conceptus cells might generate a high level of reactive oxygen species in the uterine lumen, which need to be controlled by enzymes such as catalase. A SNP close to this region was previously identified on BTA15 at $64.1 \mathrm{Mb}$ for days to first service in Brown Swiss cattle (Frischknecht et al., 2017). In addition, Peñagaricano et al. (2012) reported the presence of a SNP $251 \mathrm{~kb}$ from the $65.8 \mathrm{Mb}$ region on BTA15 that was significantly associated with sire conception rate in Holstein dairy cattle.

Two regions on BTA26 at approximately $15 \mathrm{Mb}$ associated with HCR in Holstein bulls were then validated in the analysis of SF and FertC using the heifer popula- tion. These 2 regions harbor the retinol binding protein 4 (RBP4) and myoferlin (MYOF) genes. Notably, Cole et al. (2011) identified a region close to (within 199 $\mathrm{kb}$ ) the MYOF and $R B P 4$ genes that was associated with DPR, sire calving ease, and daughter calving ease. The nuclear receptor peroxisome proliferator-activated receptor gamma (PPARG) was thought to be central for lipid metabolism and elongation of the preimplantation conceptus in dairy cows (Ribeiro et al., 2016a,b). Although no SNP within or close to PPARG was associated with fertility traits in the discovery population, SNP in PPARG were statistically associated with fertility traits of heifers (9 SNP) and cows (3 SNP). Moreover, to act as a nuclear receptor, PPARG needs to form a dimer with retinoid $\mathrm{X}$ receptor (RXR) after binding of their ligands, fatty acids, and retinol, respectively. Thus, metabolism of retinol in utero is also important for conceptus development, and $\mathrm{RBP}_{4}$ could play an essential role in bioavailability of retinol in the uterine lumen (Ribeiro et al., 2018). In fact, expression of RBP4 in the endometrium of dairy heifers increased in the period preceding conceptus elongation (Mullen et al., 2012) and, in conceptus cells, expression of RBP4 increased $94 \%$ with the onset of elongation (Ribeiro et al., 2016b).

In addition to the 7 validated SNP, the remaining 32 SNP identified in the discovery population should not be ignored. They could be validated in other populations, and this deserves further research. In fact, several genes in this list seem to be relevant for fertility. For instance, the 2 genes CUX1 (cut-like homeobox) and $B A X$ (BCL2-associated $\mathrm{X}$ protein) have strong evidence in the literature supporting their function in reproduction. In females, $B A X$ has been identified as a pro-apoptotic gene (Lazzari et al., 2011) that, when deleted, results in increased oocyte and follicle numbers in mice (Greenfeld et al., 2007). In males, however, the absence of BAX results in infertility. It has been also shown that a homozygous mutant mouse for $C U X 1$ had severely reduced male fertility (Luong et al., 2002).

Fertility traits are among the most complex, lowly heritable, and difficult to adequately measure traits. Therefore, the power of any association study to detect genetic variants related to fertility traits is probably not high. Additionally, the probability of detecting the same region in different studies becomes smaller. Also, trait definitions and strategies for editing phenotypic data, especially with respect to the handling of outlier records, differ among studies. Pryce et al. (2010) conducted a whole-genome association mapping for pregnancy rate using data from Holstein cattle and validated the results in both Holstein and Jersey populations. Notably, they could not confirm any SNP related to 
pregnancy rate in the validation populations. Visscher et al. (2012) argued that low power probably explains why these effects have not been robustly detected. Nonetheless, this indicates the challenges related to mapping and validating associations for reproduction traits and emphasizes the importance of our successful validation analyses.

In this study, different genes were validated separately for traits measured in cows and heifers. This is consistent with other genome-wide association studies in which phenotypes are separated into cow and heifer traits (Höglund et al., 2009). The correlations between SNP solutions for heifer and cow fertility traits varied from 0.29 to 0.41 . These findings agree with previous studies that investigated correlations between fertility traits measured in heifers and cows (Oltenacu et al., 1991; Jamrozik et al., 2005). Jamrozik et al. (2005) found a genetic correlation between cows and heifers of 0.60 for nonreturn rate and 0.76 for NSC in Canadian Holstein cattle. Oltenacu et al. (1991) reported a genetic correlation between heifer and cow first-service conception rate of 0.59 for Swedish Red and White. Therefore, we believe that the genetic and physiological mechanisms regulating fertility traits in heifers and cows are not essentially the same and should be studied separately.

\section{CONCLUSIONS}

The association of 1,679 SNP located within or close to 183 genes involved in lipid metabolism of conceptus cells with fertility traits in dairy cattle was investigated. A total of $39 \mathrm{SNP}$ within or close to 27 genes were significantly associated with at least one fertility trait (DPR, CCR, and HCR) in the discovery population. Seven SNP located within 6 genes (CAT, MYOF, RBP 4, GNAI1, HMOX2, and CHKA) were confirmed in the validation populations. The validated genes for fertility traits are likely important for reducing pregnancy losses. The SNP within genes confirmed in the present study are good candidates for inclusion in genomic tests of fertility traits in dairy cattle.

\section{ACKNOWLEDGMENTS}

This research was supported by the Florida Agricultural Experiment Station (Gainesville, FL), and the Natural Sciences and Engineering Research Council of Canada Discovery Grant Program (Guelph, ON, Canada). The authors thank Donald Bennink for providing the phenotypic data and the Cooperative Dairy DNA Repository (Columbia, MO) and the Council on Dairy Cattle Breeding (Bowie, MD) for facilitating the access to the genotypes.

\section{REFERENCES}

Álvarez, R., D. J. López, J. Casas, V. Lladó, M. Higuera, T. Nagy, M. Barceló, X. Busquets, and P. V. Escribá. 2015. G protein-membrane interactions I: Goil myristoyl and palmitoyl modifications in protein-lipid interactions and its implications in membrane microdomain localization. Biochim. Biophys. Acta 1851:1511-1520.

Butler, S. T. 2013. Genetic control of reproduction in dairy cows. Reprod. Fertil. Dev. 26:1-11.

Cochran, S. D., J. B. Cole, D. J. Null, and P. J. Hansen. 2013. Discovery of single nucleotide polymorphisms in candidate genes associated with fertility and production traits in Holstein cattle. BMC Genet. 14:49.

Cole, J. B., D. J. Null, and P. M. VanRaden. 2016. Phenotypic and genetic effects of recessive haplotypes on yield, longevity, and fertility. J. Dairy Sci. 99:7274-7288.

Cole, J. B., G. R. Wiggans, L. Ma, T. S. Sonstegard, T. J. Lawlor Jr., B. A. Crooker, C. P. Van Tassell, J. Yang, S. Wang, L. K. Matukumalli, and Y. Da. 2011. Genome-wide association analysis of thirty one production, health, reproduction and body conformation traits in contemporary U.S. Holstein cows. BMC Genomics $12: 408$.

Deciphering Developmental Disorders Study. 2017. Prevalence and architecture of de novo mutations in developmental disorders. Nature 542:433-438.

Durinck, S., P. T. Spellman, E. Birney, and W. Huber. 2009. Mapping identifiers for the integration of genomic datasets with the R/Bioconductor package biomaRt. Nat. Protoc. 4:1184-1191.

Frischknecht, M., B. Bapst, F. R. Seefried, H. Signer-Hasler, D. Garrick, C. Stricker, R. Fries, I. Russ, J. Sölkner, and A. Bieber. 2017. Genome-wide association studies of fertility and calving traits in Brown Swiss cattle using imputed whole-genome sequences. BMC Genomics 18:910.

Greenfeld, C. R., M. E. Pepling, J. K. Babus, P. A. Furth, and J. A. Flaws. 2007. BAX regulates follicular endowment in mice. Reproduction 133:865-876.

Han, Y., and F. Peñagaricano. 2016. Unravelling the genomic architecture of bull fertility in Holstein cattle. BMC Genet. 17:143.

Höglund, J. K., B. Guldbrandtsen, G. Su, B. Thomsen, and M. S. Lund. 2009. Genome scan detects quantitative trait loci affecting female fertility traits in Danish and Swedish Holstein cattle. J. Dairy Sci. 92:2136-2143.

Höglund, J. K., G. Sahana, R. F. Brøndum, B. Guldbrandtsen, B. Buitenhuis, and M. S. Lund. 2014. Fine mapping QTL for female fertility on BTA04 and BTA13 in dairy cattle using HD SNP and sequence data. BMC Genomics 15:790.

Holmberg, M., and L. Andersson-Eklund. 2006. Quantitative trait loci affecting fertility and calving traits in Swedish dairy cattle. J. Dairy Sci. 89:3664-3671.

Jamrozik, J., J. Fatehi, G. Kistemaker, and L. Schaeffer. 2005. Estimates of genetic parameters for Canadian Holstein female reproduction traits. J. Dairy Sci. 88:2199-2208.

Kumar, S., and U. Bandyopadhyay. 2005. Free heme toxicity and its detoxification systems in human. Toxicol. Lett. 157:175-188.

Lazzari, G., S. Colleoni, R. Duchi, A. Galli, F. D. Houghton, and C. Galli. 2011. Embryonic genotype and inbreeding affect preimplantation development in cattle. Reproduction 141:625-632.

Liu, A., Y. Wang, G. Sahana, Q. Zhang, L. Liu, M. S. Lund, and G. Su. 2017. Genome-wide association studies for female fertility traits in Chinese and Nordic Holsteins. Sci. Rep. 7:8487.

Luong, M. X., C. M. Van Der Meijden, D. Xing, R. Hesselton, E. S. Monuki, S. N. Jones, J. B. Lian, J. L. Stein, G. S. Stein, and E. J. Neufeld. 2002. Genetic ablation of the CDP/Cux protein C terminus results in hair cycle defects and reduced male fertility. Mol. Cell. Biol. 22:1424-1437.

Miller, J. K., E. Brzezinska-Slebodzinska, and F. Madsen. 1993. Oxidative stress, antioxidants, and animal function. J. Dairy Sci. 76:2812-2823

Mullen, M. P., N. Forde, M. H. Parr, M. G. Diskin, D. G. Morris, J E. Nally, A. C. O. Evans, and M. A. Crowe. 2012. Alterations in systemic concentrations of progesterone during the early luteal 
phase affect RBP4 expression in the bovine uterus. Reprod. Fertil. Dev. 24:715-722.

Muñoz-Sánchez, J., and M. E. Chánez-Cárdenas. 2014. A review on hemeoxygenase-2: Focus on cellular protection and oxygen response. Oxid. Med. Cell. Longev. 2014:604981.

Nayeri, S., M. Sargolzaei, M. Abo-Ismail, S. Miller, F. Schenkel, S. Moore, and P. Stothard. 2017. Genome-wide association study for lactation persistency, female fertility, longevity, and lifetime profit index traits in Holstein dairy cattle. J. Dairy Sci. 100:1246-1258.

Olsen, H. G., B. J. Hayes, M. P. Kent, T. Nome, M. Svendsen, A. G. Larsgard, and S. Lien. 2011. Genome-wide association mapping in Norwegian Red cattle identifies quantitative trait loci for fertility and milk production on BTA12. Anim. Genet. 42:466-474.

Oltenacu, P. A., A. Frick, and B. Lindhe. 1991. Relationship of fertility to milk yield in Swedish cattle. J. Dairy Sci. 74:264-268.

Ortega, M. S., A. C. Denicol, J. B. Cole, D. J. Null, and P. J. Hansen. 2016. Use of single nucleotide polymorphisms in candidate genes associated with daughter pregnancy rate for prediction of genetic merit for reproduction in Holstein cows. Anim. Genet. 47:288-297.

Ortega, M. S., A. C. Denicol, J. B. Cole, D. J. Null, J. F. Taylor, R. D. Schnabel, and P. J. Hansen. 2017. Association of single nucleotide polymorphisms in candidate genes previously related to genetic variation in fertility with phenotypic measurements of reproductive function in Holstein cows. J. Dairy Sci. 100:3725-3734.

Parker Gaddis, K. L., S. Dikmen, D. J. Null, J. B. Cole, and P. J. Hansen. 2017. Evaluation of genetic components in traits related to superovulation, in vitro fertilization, and embryo transfer in Holstein cattle. J. Dairy Sci. 100:2877-2891.

Peñagaricano, F., K. Weigel, and H. Khatib. 2012. Genome-wide association study identifies candidate markers for bull fertility in Holstein dairy cattle. Anim. Genet. 43:65-71.

Porto-Neto, L. R., S. Edwards, M. R. Fortes, S. A. Lehnert, A. Reverter, and M. McGowan. 2015. Genome-wide association for the outcome of fixed-time artificial insemination of Brahman heifers in northern Australia. J. Anim. Sci. 93:5119-5127.

Pryce, J. E., S. Bolormaa, A. Chamberlain, P. Bowman, K. Savin, M. Goddard, and B. Hayes. 2010. A validated genome-wide association study in 2 dairy cattle breeds for milk production and fertility traits using variable length haplotypes. J. Dairy Sci. 93:3331-3345.

Ribeiro, E. S. 2018. Symposium review: Lipids as regulators of conceptus development: Implications for metabolic regulation of reproduction in dairy cattle. J. Dairy Sci. 101:3630-3641.

Ribeiro, E. S., and M. R. Carvalho. 2017. Impact and mechanisms of inflammatory diseases on embryonic development and fertility in cattle. Anim. Reprod. 14:589-600.
Ribeiro, E. S., K. Galvão, W. Thatcher, and J. Santos. 2012. Economic aspects of applying reproductive technologies to dairy herds. Anim. Reprod. 9:370-387.

Ribeiro, E. S., L. F. Greco, R. S. Bisinotto, F. S. Lima, W. W. Thatcher, and J. E. Santos. 2016b. Biology of preimplantation conceptus at the onset of elongation in dairy cows. Biol. Reprod. 94:97.

Ribeiro, E. S., J. E. P. Santos, and W. W. Thatcher. 2016a. Role of lipids on elongation of the preimplantation conceptus in ruminants. Reproduction 152:R115-R126.

Ribeiro, E. S., J. F. W. Spricigo, M. R. Carvalho, and E. Ticiani. 2018. Physiological and cellular requirements for successful elongation of the preimplantation conceptus and the implications for fertility in lactating dairy cows. Anim. Reprod. 15:765-783.

Sahana, G., B. Guldbrandtsen, C. Bendixen, and M. S. Lund. 2010. Genome-wide association mapping for female fertility traits in Danish and Swedish Holstein cattle. Anim. Genet. 41:579-588.

Sargolzaei, M. 2014. SNP1101 User's Guide. Version 1.0. HiggsGene Solutions Inc., Guelph, Ontario, Canada.

Sargolzaei, M., J. P. Chesnais, and F. S. Schenkel. 2014. A new approach for efficient genotype imputation using information from relatives. BMC Genomics 15:478.

Schulman, N. F., G. Sahana, M. S. Lund, S. M. Viitala, and J. H. Vilkki. 2008. Quantitative trait loci for fertility traits in Finnish Ayrshire cattle. Genet. Sel. Evol. 40:195-214.

VanRaden, P., J. Cole, and K. Parker Gaddis. 2018. Net merit as a measure of lifetime profit: 2018 revision. Animal Improvement Programs Laboratory, ARS-USDA, Beltsville, MD.

Visscher, P. M., M. A. Brown, M. I. McCarthy, and J. Yang. 2012. Five years of GWAS discovery. Am. J. Hum. Genet. 90:7-24.

Wiggans, G. R., T. A. Cooper, P. M. VanRaden, C. P. Van Tassell, D. M. Bickhart, and T. S. Sonstegard. 2016. Increasing the number of single nucleotide polymorphisms used in genomic evaluation of dairy cattle. J. Dairy Sci. 99:4504-4511.

Wu, G., C. Aoyama, S. G. Young, and D. E. Vance. 2008. Early embryonic lethality caused by disruption of the gene for choline kinase $\alpha$, the first enzyme in phosphatidylcholine biosynthesis. J. Biol. Chem. 283:1456-1462.

Yang, F., M. Wang, B. Zhang, W. Xiang, K. Zhang, M. Chu, and P. Wang. 2018. Identification of new progestogen-associated networks in mammalian ovulation using bioinformatics. BMC Syst. Biol. $12: 36$. 